\title{
Biosafety Service Shared Resource
}

National Cancer Institute

\section{Source}

National Cancer Institute. Biosafety Service Shared Resource. NCI Thesaurus. Code C39331.

The Biosafety Service Shared Resource provides technical guidance and training in biological safety issues for members of Cancer Center including biological agents handling, registration of recombinant DNA and infectious agents, handling of human materials and potentially infectious materials, decontamination and disposal of biological waste, use of biosafety equipment, clean-up of biological spills. Resource assists in facilities design, advices during the construction of containment facilities, and maintains a liaison with governmental agencies regarding biosafety related matters. 